\title{
Safety and Efficacy of Phacoemulsification and Intraocular Lens Implantation in Eyes with End-Stage Chronic Angle-Closure Glaucoma
}

\author{
Seok Hwan Kim, MD ${ }^{1,2}$, Young Keun Han, MD ${ }^{1,2}$ \\ 'Department of Ophthalmology, Seoul National University College of Medicine, Seoul, Korea \\ ²Department of Ophthalmology, Seoul National University Boramae Hospital, Seoul, Korea
}

\begin{abstract}
Purpose: To evaluate surgical outcomes of phacoemulsification and intraocular lens implantation in eyes with end-stage chronic angle-closure glaucoma (CACG).

Methods: Medical records of patients with end-stage CACG (mean deviation, $<-19 \mathrm{~dB}$ ) and clinically significant cataracts who underwent phacoemulsification and intraocular lens implantation were retrospectively reviewed. During at least 12 months of follow-up, changes in visual acuity, intraocular pressure, visual field parameters, and number of antiglaucoma agents used were assessed. Intraoperative and postoperative complications were documented. Multiple regression analysis was performed to identify independent predictors of postoperative visual outcome.

Results: Forty-two eyes of 38 subjects were enrolled. The mean follow-up period was 19.7 months (12-33 months). Corrected visual acuity improved from $0.63 \pm 0.53$ logarithm of the minimum angle of resolution (logMAR) preoperatively to $0.21 \pm 0.33 \log M A R$ postoperatively $(p<0.001)$. Intraocular pressure decreased from $15.50 \pm 3.23 \mathrm{mmHg}$ to $11.05 \pm 1.76 \mathrm{mmHg}(p<0.001)$. No change was found in visual field parameters or number of antiglaucoma agents used. During surgery, 4 eyes had zonular dialysis and 1 eye had posterior capsule rupture. After surgery, 2 eye required additional trabeculectomy due to uncontrolled intraocular pressure after phacoemulsification. Preoperative visual acuity and mean sensitivities of 4 central points of the visual field were independent predictors of postoperative visual outcome.
\end{abstract}

Conclusions: Phacoemulsification and intraocular lens implantation was performed safely and effectively in end-stage CACG eyes with cataracts.

Key words: Phacoemulsification; Cataract; Chronic angle-closure glaucoma; End-stage glaucoma

\section{Introduction}

Chronic angle-closure glaucoma (CACG), in which closure of the anterior chamber angle leads to increased intraocular pressure (IOP) and glaucomatous optic neuropathy, is a leading cause of blindness throughout the world. $^{1-4} \mathrm{~A}$ thick and anteriorly positioned crystalline lens plays a pivotal role in the pathogenesis of CACG. ${ }^{5}$ There-

Received: 2015. 9. 20.

Revised: 2015. 10. 28.

Accepted: 2015. 11. 11.

Corresponding Author: Seok Hwan Kim , MD

Department of Ophthalmology, Seoul National University Boramae

Hospital, 20 Boramae-ro 5-gil, Dongjak-gu, Seoul 07061, Korea

Tel: +82-2-870-2415, Fax: +82-2-831-2826

E-mail: xcski@hanmail.net

* None of the authors have any financial/conflicting interests to disclose. fore, lens extraction itself has a favorable outcome for the control of IOP and vision improvement in eyes with CACG and cataracts. ${ }^{6,7}$ Recent studies have shown that results of phacoemulsification alone were comparable to those of combined phacoemulsification and trabeculectomy in terms of IOP control in eyes with CACG and cataracts. ${ }^{8,9}$

In cases of advanced glaucoma with a visually significant cataract, combined phacoemulsification and trabeculectomy is usually advocated because uncontrolled IOP after cataract extraction alone carries a risk of postoperative visual field loss, which may involve fixation with central vision loss. ${ }^{10}$ However, trabeculectomy in end-stage glaucoma carries a risk of sudden vision loss ("wipe-out phenomenon") as well as vision-threatening complications like prolonged hypotony, choroidal detachment, and malignant glaucoma. ${ }^{11-13}$ In addition, combined surgery requires additional skill and 
a longer surgical duration, and may have prolonged visual recovery. Therefore, in eyes with end-stage CACG and cataracts, phacoemulsification alone might be an alternative to conventional combined surgery because phacoemulsification can be expected to reduce IOP in eyes with CACG.

In this study, we evaluated the safety and efficacy of phacoemulsification and intraocular lens implantation in terms of visual outcome and IOP in eyes with end-stage CACG and cataracts.

\section{Patients and Methods}

Medical records of patients who had end-stage CACG and clinically significant cataracts, and underwent phacoemulsification between August 2008 and July 2012 at Boramae Medical Center were retrospectively reviewed. The patients with at least 12 months of postoperative follow-up were included. This study was approved by the institutional review board of Boramae Medical Center.

CACG was defined by the presence of all of the following factors: glaucomatous optic neuropathy with compatible visual field defects, 2 or more quadrants of angle-closure obscuring the pigmented part of the trabecular meshwork under gonioscopy in the presence of patent laser iridotomy, and IOP $>21 \mathrm{mmHg}$ without use of antiglaucoma medications. ${ }^{8}$ End-stage glaucoma was defined on the basis of visual field results if the mean deviation (MD) on Humphrey automated perimetry 24-2 (Carl Zeiss Meditec, Inc., Dublin, CA) was $-19 \mathrm{~dB}$ or worse. ${ }^{14}$ Eyes with a clinically significant cataract and visual acuity of 30/50 or worse were included. Eyes with a history of intraocular surgery, except laser iridotomy and laser peripheral iridoplasty, were excluded. Eyes with secondary angle-closure glaucoma were excluded. We also excluded all eyes with macular disease or other intraocular eye diseases affecting the visual field (e.g., age-related macular degeneration, diabetic retinopathy, retinal vein occlusion, and optic neuropathy other than glaucoma). Only patients with end-stage CACG who had an IOP of $\leq 25 \mathrm{mmHg}$ with use of 3 or fewer topical drugs were included in this study because patients with end-stage CACG and cataract who had an IOP of $>25 \mathrm{mmHg}$ despite topical medications underwent combined cataract surgery and trabeculectomy.

A preoperative medical history was obtained for determining presence of previous intraocular surgery, coexisting systemic disease and use of systemic medications. A baseline ophthalmic examination was performed within 3 days before surgery; this examination included a visual acuity test, manifest refraction and correction, IOP measurements by Goldmann applanation tonometry, slit-lamp examination, gonioscopy, and fundus examination. Humphrey Swedish interactive thresholding algorithm (SITA) 24-2 perimetry and optical biometer (IOLMaster, Carl Zeiss Meditec, Inc., Dublin, CA) were performed. The number of preoperative antiglaucoma medications used by the patient was documented.

Patients were examined postoperatively at 1 day, 1 week, 1 month, 3 months, and 6 months and thereafter, at an interval of 3 to 6 months; the examination included assessment of best-corrected visual acuity, IOP, intraocular lens status, and the number of antiglaucoma agents that was required to achieve an optimal IOP level. Additional visits were scheduled as clinically warranted. The incidence of intraoperative and postoperative complications such as posterior capsular rupture, zonular dialysis, and IOP spike (IOP > $21 \mathrm{mmHg}$ ) were documented at each visit. Assessment of visual fields was repeated at an interval of 6 months after surgery.

Main outcome measures included the change in logarithm of the minimum angle of resolution $(\log$ MAR) and IOP. The MD and mean sensitivity of the 4 central visual field points in the latest assessment of the visual field after surgery were compared with preoperative values. Factors related to the final visual acuity outcome were also identified.

\section{Surgical technique}

The surgical procedure was standard for all subjects, and all procedures were performed by 1 experienced surgeon (SH Kim). Phacoemulsification was performed using topical or sub-Tenon anesthesia of 2\% lidocaine. A 3.2-mm clear corneal incision was placed temporally. After instillation of $1.0 \%$ sodium hyaluronate into the anterior chamber, a continuous curvilinear capsulorhexis was created with a bent 
27-gauge needle. Hydrodissection and phacoemulsification of the nucleus was performed using the stop-and-chop technique, and the residual cortex was subsequently removed. A foldable acrylic intraocular lens (Bausch \& Lomb; Akreos Adapt, New Jersey, USA) was placed, and the viscoelastic material was aggressively evacuated from the anterior chamber to avoid an immediate postoperative increase in the IOP. The intraocular lens was implanted in the capsular bag in all but 1 patient, in whom the intraocular lens was placed with scleral fixation because of severe zonular dialysis and posterior capsule rupture. In an eye with zonular dialysis less than $90^{\circ}$, the intraocular lens was placed in the capsular bag after a capsular tension ring was inserted to stabilize zonular tension. To prevent a postoperative IOP spike, 1 drop of $0.5 \%$ timolol maleate was applied immediately after cataract surgery. Use of antiglaucoma agents was continued for the patients on the day of surgery.

\section{Statistical analysis}

A paired t-test was performed to compare preoperative and postoperative values of visual acuity, IOP, number of antiglaucoma agents used, MD, and the mean sensitivity of the 4 central visual field points. Multiple regression analysis was performed to identify independent predictors of postoperative visual outcome. The correlation between postoperative visual acuity and preoperative variables was analyzed using the Pearson correlation. Variables with a $p$ value less than 0.05 were included in a backward stepwise multiple linear regression after assessment of collinearity. Statistical analysis was performed using SPSS version 18.0 (SPSS Inc., Chicago, IL, USA). A $p$ value of less than 0.05 was accepted as statistically significant.

\section{Results}

Initially, 47 eyes of 43 patients who were diagnosed with end-stage CACG and clinically significant cataracts, and underwent phacoemulsification were enrolled. Among selected 47 eyes, 5 eyes of 5 patients were excluded because of follow-up loss. Finally, the study involved 42 eyes of 38 patients. Table 1 shows the patients' demographic characteristics. The mean age of patients at the time of surgery

Table 1. Demographic features of 42 eyes of 38 patients with end-stage chronic angle-closure glaucoma and cataract who underwent phacoemulsification and intraocular lens implantation

\begin{tabular}{lc}
\hline \multicolumn{1}{c}{ Parameters } & Values \\
\hline Age (years, mean \pm SD) & $74.37 \pm 5.81$ (range: 65 to 86 years) \\
Gender (male:female) & $14: 24$ \\
Right eye : Left eye & $19: 23$ \\
Follow-up (months, mean \pm SD) & $19.68 \pm 7.89$ (range: 12 to 33 months) \\
Extent of synechial angle closure & $291.4 \pm 71.0$ (range: 180 to 360 ) \\
Eyes with history of acute angle closure attack & $12 / 42$
\end{tabular}

$\mathrm{SD}=$ standard deviation

Table 2. Preoperative and postoperative clinical status of 42 eyes of 38 patients with end-stage chronic angle-closure glaucoma and cataract who underwent phacoemulsification and intraocular lens implantation

\begin{tabular}{lcrc}
\hline & $\begin{array}{c}\text { Baseline } \\
\text { (mean } \pm \text { SD) }\end{array}$ & $\begin{array}{c}\text { Final visit } \\
\text { (mean } \pm \text { SD) }\end{array}$ & $p$-value* \\
\hline IOP (mmHg) & $15.50 \pm 3.23$ & $11.05 \pm 1.76$ & $<0.001$ \\
Visual acuity (logMAR) & $0.63 \pm 0.53$ & $0.21 \pm 0.33$ & $<0.001$ \\
Mean deviation (dB) & $-26.08 \pm 3.23$ & $-25.87 \pm 3.34$ & 0.516 \\
Mean sensitivity of 4 central visual field points (dB) & $11.92 \pm 7.72$ & $12.15 \pm 6.75$ & 0.622 \\
Number of medications & $1.95 \pm 1.09$ & $1.95 \pm 0.72$ & 0.999
\end{tabular}

*Paired $t$-test. 


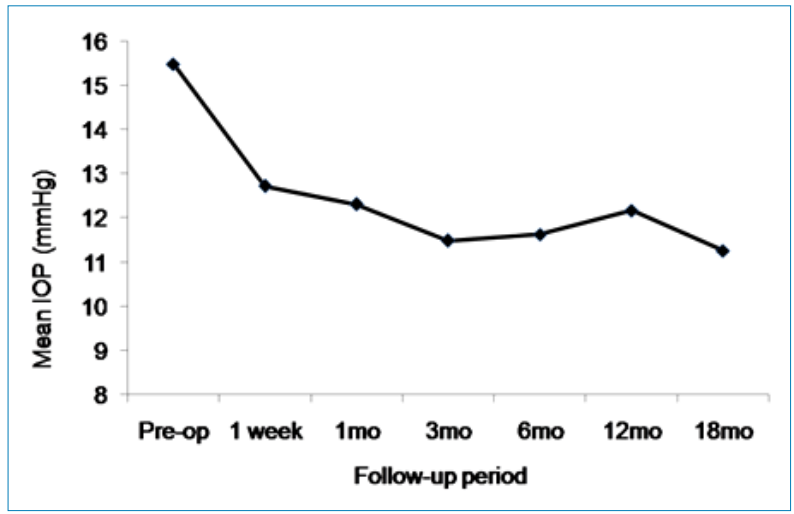

Figure 1. Intraocular pressure changes after phacoemulsification and intraocular lens implantation in 42 eyes with end-stage chronic angle-closure glaucoma and cataract. Mo, month.

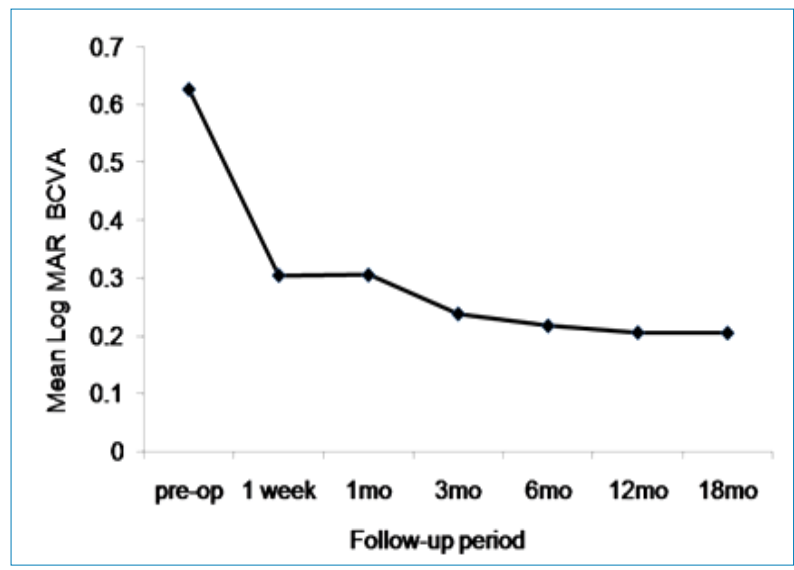

Figure 2. Log MAR BCVA (best corrected visual acuity) changes after phacoemulsification and intraocular lens implantation in 42 eyes with end-stage chronic angle-closure glaucoma and cataract. mo, month.

was $74.37 \pm 5.81$ years (range: 65 to 86 years). The mean follow-up period was $19.68 \pm 7.89$ (range: 12 to 33 months). No eyes had mature or white cataracts.

Table 2 shows preoperative and postoperative clinical status of 42 eyes. We observed a significant reduction in IOP from $15.50 \pm 3.23 \mathrm{mmHg}$ (range: 11 to $23 \mathrm{mmHg}$ ) to $11.05 \pm$ $1.76 \mathrm{mmHg}$ (range: 8 to $16 \mathrm{mmHg}$ ). (Fig. 1) The mean logMAR visual acuity was also significantly improved from $0.63 \pm 0.53$ to $0.21 \pm 0.33$ after phacoemulsification. (Fig. 2) Significant changes were not observed in MD, the mean sensitivity of the 4 central visual field points, or the number of antiglaucoma agents used.

Table 3 lists intraoperative and postoperative compli-
Table 3. Intraoperative and postoperative complications of phacoemulsification and intraocular lens implantation in 42 eyes with end-stage chronic angle-closure glaucoma

\begin{tabular}{lcr}
\hline Complications & Eyes & Rate \\
\hline Intraoperative & & \\
$\quad$ Posterior capsule tear & 1 & $2.4 \%$ \\
$\quad$ Zonular dialysis & 4 & $9.5 \%$ \\
$\quad$ Iris synechiolysis & 7 & $16.7 \%$ \\
Postoperative & & \\
Uncontrolled IOP & 2 & $4.8 \%$ \\
Anterior uveitis & 2 & $4.8 \%$ \\
\hline
\end{tabular}

cations. During surgery, zonular dialysis was found in 4 eyes. Among 4 eyes, three eyes had capsular tension ring implantation because zonular dialysis of less than $90^{\circ}$ was identified during cataract surgery. The other eye had anterior vitrectomy and scleral fixation of the intraocular lens due to severe zonular dialysis and posterior capsule rupture. For this eye, intravenous mannitol was administered immediately after surgery to prevent a postoperative IOP spike. After surgery, 2 eyes showed severe anterior chamber inflammation with fibrin exudate, which was controlled by frequent application of topical $1 \%$ prednisolone acetate. Two eyes of 2 patients showed IOP elevation despite use of antiglaucoma agents at 1 month and at 2 months respectively after cataract surgery, and the patients underwent additional trabeculectomy. No immediate IOP spike was observed 1 day after cataract surgery in all patients.

In multiple linear regression analysis, preoperative logMAR visual acuity and preoperative mean sensitivity of the 4 central visual field points were identified as independent predictors of postoperative $\log$ MAR visual acuity after adjusting for preoperative IOP and MD $\left(\mathrm{R}^{2}=0.649, p<\right.$ 0.001).

\section{Discussion}

This retrospective case series suggests that phacoemulsification and intraocular lens implantation is a safe and effective option for the treatment of eyes with end-stage CACG and cataracts. We also showed that preoperative vision and the mean sensitivity of the 4 central points of the visual field were independent predictors of postoperative visual 
outcome.

Combined cataract surgery and trabeculectomy is usually advocated for eyes with advanced glaucoma and clinically significant cataracts. ${ }^{10}$ The advantages of combined surgery includes the patients' convenience of undergoing 2 surgeries on 1 day, the apparent IOP-lowering effect and reduced requirement of antiglaucoma medications due to trabeculectomy, and prevention of the postoperative IOP spike associated with cataract surgery. However, combined surgery has several disadvantages. Combined surgery has more complications than cataract surgery alone, such as postoperative hypotony, hyphema, choroidal detachment, and malignant glaucoma. ${ }^{11-13}$ Although most of these complications are transient and treatable, they hamper early visual rehabilitation and might induce permanent vision loss. Trabeculectomy in end-stage glaucoma also carries the risk of sudden, unexplained vision loss ("wipe-out phenomenon"), although it is very rare. ${ }^{13,15}$ In addition, combined surgery requires additional skill for trabeculectomy, longer surgical duration, more follow-up, and additional surgical intervention in the postoperative period to maintain bleb function and to manage complications. ${ }^{8}$ Recent studies showed that the results of phacoemulsification alone were comparable to those of combined phacoemulsification and trabeculectomy for the control of IOP in eyes with CACG and cataracts. ${ }^{8,9}$ In this study, we showed that for eyes with end-stage CACG, phacoemulsification alone provides favorable visual outcome and control of IOP. Because this study was a case series, further studies comparing the outcome between phacoemulsification alone and combined surgery should be performed for eyes with end-stage CACG. Furthermore, since we included eyes with relatively medically controlled CACG and cataracts in this study, additional studies are warranted for eyes with medically uncontrolled end-stage CACG and cataracts.

Previous reports have showed that in eyes with CACG, cataract surgery reduced the number of antiglaucoma medications used as well as IOP, ${ }^{8,9,16}$ in contrast, in the present study, there was no difference in the number of antiglaucoma agents used preoperatively and postoperatively. This can be partly explained by the end-stage visual field status of the study subjects. Given the possibility of an IOP spike after discontinuation of antiglaucoma agents, which might involve fixation with central vision loss, the author hesitated to discontinue antiglaucoma agents despite good IOP control.

Only 2 eye among 42 eyes required additional trabeculectomy to control IOP after cataract surgery alone in this study. IOPs of both eyes were well controlled after trabeculectomy without any complications. Our results imply that additional trabeculectomy can be performed successfully in cases with uncontrolled IOP after performing phacoemulsification alone.

An early postoperative IOP spike can be minimized with topical or systemic antiglaucoma medications. ${ }^{17,18} \mathrm{~A}$ recent randomized clinical trial reported that immediate postoperative treatment with $0.5 \%$ timolol maleate reduced the occurrence of IOP spikes. ${ }^{19}$ In this study, we applied $0.5 \%$ timolol maleate immediately after phacoemulsification to prevent an early postoperative IOP spike. In addition, we did not discontinue antiglaucoma agents even on the day of surgery, and we judiciously removed viscoelastic agents after intraocular lens implantation. Prophylactic intravenous mannitol was administered in the case of an eye with posterior capsule rupture and scleral fixation of the intraocular lens; this eye had a high chance of an IOP spike. Among 42 eyes in this study, none showed IOP elevation at 1 day or 1 week after surgery.

In eyes with end-stage glaucoma and a coexisting cataract, visual outcome is often difficult to predict after cataract surgery because glaucoma also contributes to the vision decrease. Herein, we performed multiple regression analysis and found that preoperative vision and the mean sensitivity of 4 central points of the visual field were independent predictors of postoperative visual outcome.

Our study does not suggest that phacoemulsification alone should be a substitute for combined surgery for the treatment of eyes with end-stage CACG and cataracts. We found that the IOP-lowering effect of phacoemulsification was modest (mean, $4.5 \mathrm{mmHg}$ ) and that the number of antiglaucoma agents used was not different postoperatively. Therefore, the risks and benefits of combined surgery should be 
weighed carefully against those of cataract surgery alone for the treatment of eyes with end-stage CACG. In addition, patients should be informed preoperatively that an additional trabeculectomy may be required.

In conclusion, this study showed that phacoemulsification and intraocular lens implantation can be performed safely and effectively in eyes with medically controlled end-stage CACG. Further studies comparing the outcome between phacoemulsification alone and combined surgery in endstage CACG should be warranted.

\section{References}

1. Foster PJ. The epidemiology of primary angle closure and associated glaucomatous optic neuropathy. Semin Ophthalmol 2002; 17:50-8.

2. Foster PJ, Baasanhu J, Alsbirk PH, et al. Glaucoma in Mongolia. A population-based survey in Hövsgöl province, northern Mongolia. Arch Ophthalmol 1996;114:1235-41.

3. Johnson GJ, Foster PJ. Can we prevent angle-closure glaucoma? Eye. 2005;19:1119-24.

4. Dandona L, Dandona R, Mandal P, et al. Angle-closure glaucoma in an urban population in southern India. The Andhra Pradesh eye disease study. Ophthalmology. 2000;107:1710-6.

5. Tarongoy P, Ho CL, Walton DS. Angle-closure glaucoma: the role of the lens in the pathogenesis, prevention, and treatment. Surv Ophthalmol 2009;54:211-25.

6. Hayashi K, Hayashi H, Nakao F, Hayashi F. Effect of cataract surgery on intraocular pressure control in glaucoma patients. J Cataract Refract Surg 2001;27:1779-86.

7. Yang $\mathrm{CH}$, Hung PT. Intraocular lens position and anterior chamber angle changes after cataract extraction in eyes with primary angle-closure glaucoma. J Cataract Refract Surg 1997;23:1109-13.

8. Tham CC, Kwong YY, Leung DY, et al. Phacoemulsification versus combined phacotrabeculectomy in medically controlled chronic angle closure glaucoma with cataract.
Ophthalmology 2008;115:2167-73

9. Tham CC, Kwong YY, Leung DY, et al. Phacoemulsification versus combined phacotrabeculectomy in medically uncontrolled chronic angle closure glaucoma with cataracts. Ophthalmology 2009;116:725-31.

10. Khatana AK, Cohen JS, Osher RH. Combined cataract implant and filtering surgery. In:Steinert RF, ed, Cataract Surgery. Maryland Heights, Elsevier, Inc.; 2010; 259-81

11. Bellucci R, Perfetti S, Babighian S, et al. Filtration and complications after trabeculectomy and after phaco-trabeculectomy. Acta Ophthalmol Scand Suppl 1997;224:445.

12. Law SK, Nguyen AM, Coleman AL, Caprioli J. Severe loss of central vision in patients with advanced glaucoma undergoing trabeculectomy. Arch Ophthalmol 2007; 125:1044-50.

13. Costa VP, Smith M, Spaeth GL, et al. Loss of visual acuity after trabeculectomy. Ophthalmology 1993;100:599-612.

14. Much JW, Liu C, Piltz-Seymour JR. Long-term survival of central visual field in end-stage glaucoma. Ophthalmology 2008;115:1162-6.

15. Aggarwal SP, Hendeles S. Risk of sudden visual loss following trabeculectomy in advanced primary open-angle glaucoma. Br J Ophthalmol 1986 ;70:97-9.

16. Shrivastava A, Singh K. The effect of cataract extraction on intraocular pressure. Curr Opin Ophthalmol 2010 ;21:118-22.

17. Fry LL. Comparison of the postoperative intraocular pressure with Betagan, Betoptic, Timoptic, Iopidine, Diamox, Pilopine Gel, and Miostat. J Cataract Refract Surg 1992;18:14-9.

18. Haimann MH, Phelps CD. Prophylactic timolol for the prevention of high intraocular pressure after cataract extraction. A randomized, prospective, double-blind trial. Ophthalmology 1981;88:233-8.

19. Levkovitch-Verbin H, Habot-Wilner Z, Burla N, et al. Intraocular pressure elevation within the first 24 hours after cataract surgery in patients with glaucoma or exfoliation syndrome. Ophthalmology 2008;115:104-8. 